\title{
Aprendizaje Basado en Retos aplicado al desarrollo de tesis profesionales: Caso Ingeniería en Ciencias de la Computación
}

\section{Challenge Based Learning applied to the development of professional theses: Case Engineering in Computer Science}

\author{
DOI: $10.46932 / \mathrm{sfjdv2n2-127}$
}

Received in: March 1st, 2021

Accepted in: May 30th, 2021

\section{Dr. Jose Luir Hernandez Ameca}

Facultad de Ciencias de la Computación, Benemérita Universidad autónoma de Puebla (BUAP). Av. San Claudio y 14 Sur Ciudad Universitaria C.P. 72570.Puebla, Puebla. México. mecajl@hotmail.com

\section{M.I. María del Consuelo Molina García}

Facultad de Ciencias de la Computación, Benemérita Universidad autónoma de Puebla (BUAP). Av. San Claudio y 14 Sur Ciudad Universitaria C.P. 72570.Puebla, Puebla. México.

E-mail: chelito.molina@gmail.com

\section{Cinthya Karla Saldaña Escalona}

Benemérita Universidad Autónoma De Puebla (BUAP), Facultad de Ciencias de la Computación, Benemérita Universidad autónoma de Puebla (BUAP).

Av. San Claudio y 14 Sur Ciudad Universitaria C.P. 72570.Puebla, Puebla. México.

E-mail: cinthya_escalona@outlook.com

\section{María Del Carmen Báez Salazar}

Benemérita Universidad Autónoma De Puebla (BUAP), Facultad de Ciencias de la Computación, Benemérita Universidad autónoma de Puebla (BUAP). Av. San Claudio y 14 Sur Ciudad Universitaria C.P. 72570.Puebla, Puebla. México.

E-mail: mcarmen.basa05@gmail.com

\section{RESUMEN}

La presente investigación tiene como objetivo utilizar la metodología de Aprendizaje Basado en Retos (ABR) como una estrategia para incentivar la realización de tesis de licenciatura en la carrera de Ingeniería en Ciencias de la Computación de la Benemérita Universidad Autónoma de Puebla. Dentro de dicha facultad el Laboratorio de Sistemas Robóticos ofertó temas de tesis como retos tecnológicos orientados al medioambiente y la salud, basando su desarrollo en la metodología ABR a partir del año 2017. Se aplicaron a los alumnos titulados encuestas para medir el uso y aplicación de: conocimientos teóricoprácticos, habilidades y competencias transversales, durante el desarrollo de la tesis. Los resultados mostraron que el alumno desarrollo y aplico conocimientos, habilidades y competencias de una forma natural, el número de tesistas que concluyeron su trabajo de investigación aumento de uno a seis. Por lo que se concluye que la metodología funciona óptimamente, en función del compromiso alumno-asesor, se propone seguir aplicándola para mejorarla y aumentar la calidad de sus productos.

Palabras clave: Aprendizaje Basado en Retos, Ciencias de la Computación, Competencias, Tesis. 


\begin{abstract}
This research aims to use the methodology of Challenge based learning (CBL) as a strategy to encourage the realization of a bachelor's thesis in the Computer Science Engineering degree at the Benemérita Universidad Autónoma de Puebla. Within this faculty, the Robotics Systems Laboratory offered thesis topics such as technological challenges oriented to the environment and health, basing its development on the ABR methodology as of 2017. Surveys were applied to students entitled to measure the use and application of : theoretical-practical knowledge, skills and transversal skills, during the development of the thesis. The results showed that the student developed and applied knowledge, skills and competences in a natural way, the number of thesis students who concluded their research work increased from one to six. Therefore, it is concluded that the methodology works optimally, depending on the student-advisor commitment, it is proposed to continue applying it to improve it and increase the quality of its products.
\end{abstract}

Key words: Challenge based learning, Computer Science, Skills, Thesis

\title{
1 INTRODUCCIÓN
}

El perfil de egreso de los alumnos de la Ingeniería en Ciencias de la Computación (ICC) especifica el dominio de competencias que les permitan desarrollarse en empresas, institutos de investigación o continuar un posgrado [1]. Los problemas que actualmente enfrentan los ingenieros poseen dimensiones sociales, tecnológicas y económicas. Se concluye que el ingeniero necesita: saber pensar, saber desempeñar, saber interpretar y saber actuar [2]. Actualmente las empresas son más selectivas respecto al talento que eligen "queremos jóvenes que entiendan cómo se trabaja con una cultura abierta, toma de decisiones, responsabilidades y dar el extra" [3]. En Bélgica se ha desarrollado e implementado una metodología basada en desafíos; se reportan resultados y aprendizajes, que generan pasantías, tesis de maestría y propuestas de trabajo. Por dichas razones se propone aplicar el Aprendizaje Basado en Retos (ABR) para el desarrollo de tesis de grado dentro de la Ingeniería en Ciencias de la Computación, donde los estudiantes desarrollan soluciones a problemas reales que requieren un abordaje interdisciplinario y creativo. Este trabajo reporta la evaluación de los resultados obtenidos considerando la integración de contenidos teóricos y prácticos, el uso individual de conocimientos, habilidades, competencias transversales, motivación y tiempo.

\section{DESARROLLO}

El ABR tiene sus raíces en el Aprendizaje Vivencial, el cual tiene como principio fundamental que los estudiantes aprenden mejor cuando participan de forma activa en experiencias abiertas de aprendizaje, qué cuando participan de manera pasiva en actividades estructuradas [4]. Este enfoque práctico, tiene como propósito promover en los estudiantes un conocimiento más profundo, identificar y resolver retos en sus comunidades, así como compartir los resultados con el mundo. Proporciona problemas suficientemente grandes para aprender nuevas ideas y herramientas para resolverlos, pero a la vez, lo 
suficientemente cercanos que incentivan el hallazgo de una solución [5]. En el presente trabajo se investigan los efectos de utilizar la metodología ABR, en el desarrollo de tesis de grado como parte de una estrategia que busca integrar conocimientos teórico-prácticos, incentivar habilidades y desarrollar competencias transversales. La investigación se realiza dentro del Laboratorio de Sistemas Robóticos (SIRO) de la Facultad de Ciencias de la Computación de la Benemérita Universidad Autónoma de Puebla (BUAP), de 2016 a 2018. Se aplicó la metodología de investigación transeccional, donde se evaluó la aplicación de variables cualitativas (conocimientos teórico-prácticos, habilidades y competencias transversales) y analizó cuantitativamente su incidencia por parte del estudiante al finalizar la tesis.

\subsection{MARCO TEÓRICO}

Actualmente, los estudiantes acceden a la información de una forma sustancialmente distinta a la de hace algunos años. Regulan mucho de su conocimiento a través de un aprendizaje informal y han pasado de ser consumidores de información, a productores de la misma. Como resultado, los métodos tradicionales de enseñanza-aprendizaje están siendo cada vez menos efectivos para atraer a los estudiantes y motivarlos a aprender [6]. El ABR es un enfoque pedagógico que se ha incorporado en áreas de estudio como la ciencia y la ingeniería, y demanda una perspectiva del mundo real porque sugiere que el aprendizaje involucra el hacer o actuar del estudiante respecto a un tema de estudio [7]. El ABR aprovecha el interés de los estudiantes por darle un significado práctico a la educación, mientras desarrollan competencias claves como el trabajo colaborativo y multidisciplinario, la toma de decisiones, la comunicación avanzada, la ética y el liderazgo [8]. Tiene como propósito promover un conocimiento más profundo de los contenidos que se están estudiando, identificar y resolver retos en sus comunidades, así como compartir los resultados con el mundo. Este modelo hace relevante el aprendizaje, pues da a los estudiantes problemas suficientemente grandes para aprender nuevas ideas y herramientas para resolverlos, pero a la vez, lo suficientemente cercanos para que les sea importante encontrar una solución [9]. La Escuela de Medicina y Ciencias de la Salud del Tecnológico de Monterrey utilizo el ABR para realizar la Semana i: Retos de innovación enfrentando problemas de salud, donde el objetivo de este estudio fue evaluar las competencias de pensamiento crítico y creativo. Esta investigación incorporó un abordaje cuantitativo, mediante un estudio descriptivo y transeccional [10]. Estudiantes de ingeniera del Tecnológico de Monterrey utilizaron el ABR en la materia de Informática Industrial, como reto se les propuso participar en una competencia de robótica, como resultados reportan en los alumnos el aumento de la motivación de aprendizaje, innovación y trabajo en equipo [11]. En la universidad de Cincinnati, se utilizó el ABR en el curso de circuitos en la licenciatura de Electrónica donde se propusieron diferentes 
retos generales con lo cual se logró reducir experiencias negativas y aumentar la motivación de los estudiantes.

El Laboratorio SIRO tiene la misión de contribuir con la sociedad y el medio ambiente proponiendo e implementando soluciones, utilizando las ciencias de la computación, en particular la robótica. Para cumplir sus objetivos se han generado proyectos (de investigación-innovación y tesis), integradores de conocimientos teórico-prácticos, habilidades y competencias transversales. En este trabajo se reportan los resultados de aplicar el ABR en el desarrollo y conclusión de Tesis de grado.

\subsection{PLANTEAMIENTO DEL PROBLEMA}

De acuerdo a estadísticas internas se ha detectado que los alumnos de la carrera de ICC en la actualidad no están motivados a titularse por medio de la realización de tesis, siéndoles más atractivo otras opciones de titulación, por ejemplo: titulación automática, examen Egel-Ceneval, por diplomado y experiencia profesional. Además, los alumnos no son conscientes de las competencias que han desarrollado a lo largo de la carrera por lo cual no se sienten capaces de comprometerse a concluir un proyecto de investigación.

En base al problema anteriormente mencionado, se muestran los resultados de aplicar el ABR como una metodología funcional en la motivación de los estudiantes para realizar un trabajo de tesis.

\subsection{MÉTODO}

En base a la metodología ABR se propuso el modelo de trabajo que se muestra en la figura 1 
Figura 1: Modelo de trabajo propuesto en base a la metodología ABR.

investigación

El reto

- El reto nace de la detección de problemas relacionados con la salud, el medio ambiente y la educación.

- La investigación se realiza a partir de las preguntas generadoras que el asesor le da al alumno, el alumno busca respuestas desde su formación profesional como ingeniero en ciencias de la computación.

- La solución se propone de acuerdo a criterios de viabilidad, supervisados por el asesor.

- La implementación y la evaluación se realizan utilizando una tecnología específica aplicando los conocimientos teórico-prácticos, habilidades y competencias transversales, y mediante un instrumento de evaluación compara los resultados obtenidos contra los esperados.

- La reflexión y publicación se ve reflejada en la escritura del trabajo de tesis en base a un formato específico.

\subsection{RESULTADOS}

En 2016 el SIRO contaba con tres alumnos realizando su tesis, llegando a la culminación solo uno de ellos, durante ese año no se contaba con una metodología especifica de trabajo lo cual genero diversos problemas como la falta de planeación, motivación y compromiso de trabajo por parte del asesor y el alumno. Del 2017 al 2018 el número total de titulados incremento de uno a seis, en 2017 se titularon dos alumnos y en 2018 cuatro de ellos, durante dicho periodo se implementó por parte del asesor la metodología ABR con lo cual la planeación, motivación y compromiso de ambas partes incremento. 
Una vez concluido y defendido el trabajo de tesis, se aplicaron tres encuestas a cada uno de los seis titulados con la finalidad de medir su percepción sobre la aplicación de (conocimientos teóricoprácticos), (habilidades) y (competencias transversales) durante el desarrollo de la tesis. Las encuestas se basaron en una escala tipo Likert de 3 niveles (nada, poco, mucho), los resultados obtenidos se muestran en las figuras 2 a 4.

Figura 2: Resultaos de la encuesta (aplicación de los conocimientos teórico-prácticos).

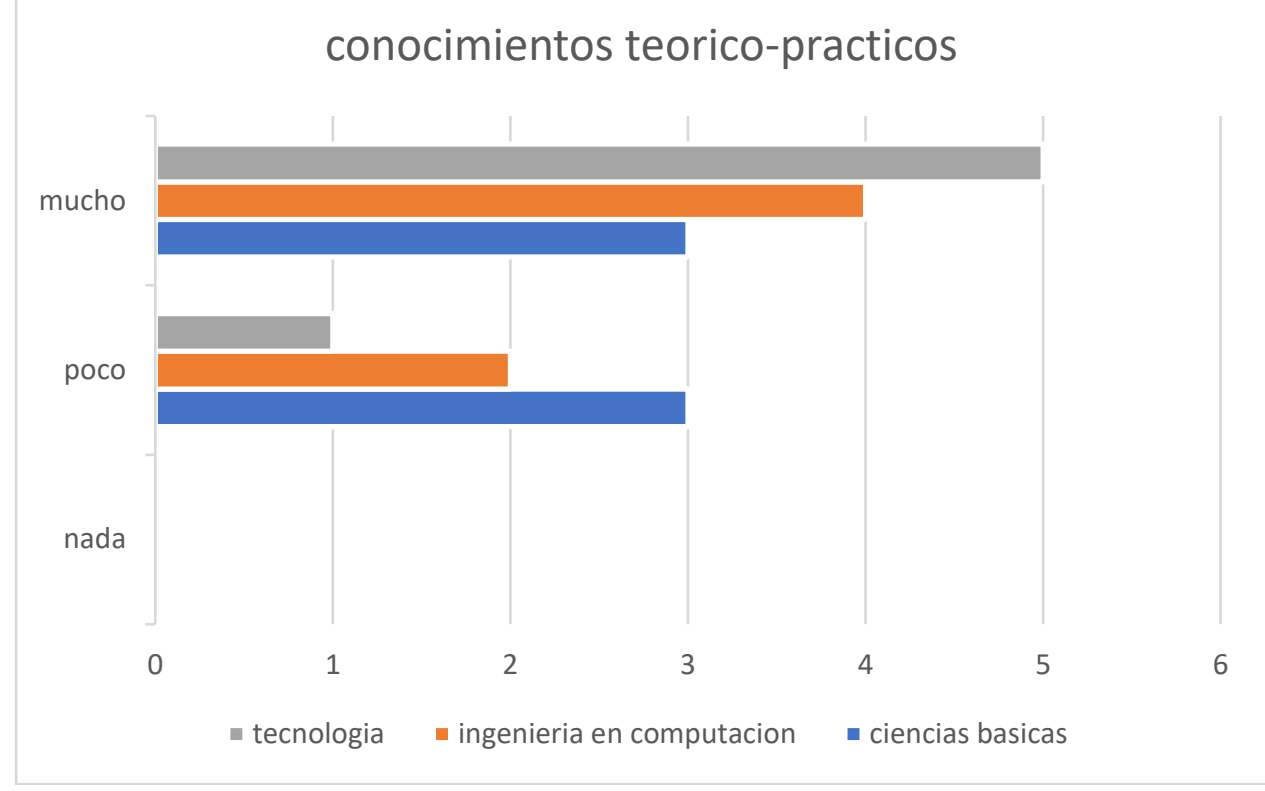

Figura 3: Resultaos de la encuesta (aplicación de habilidades).

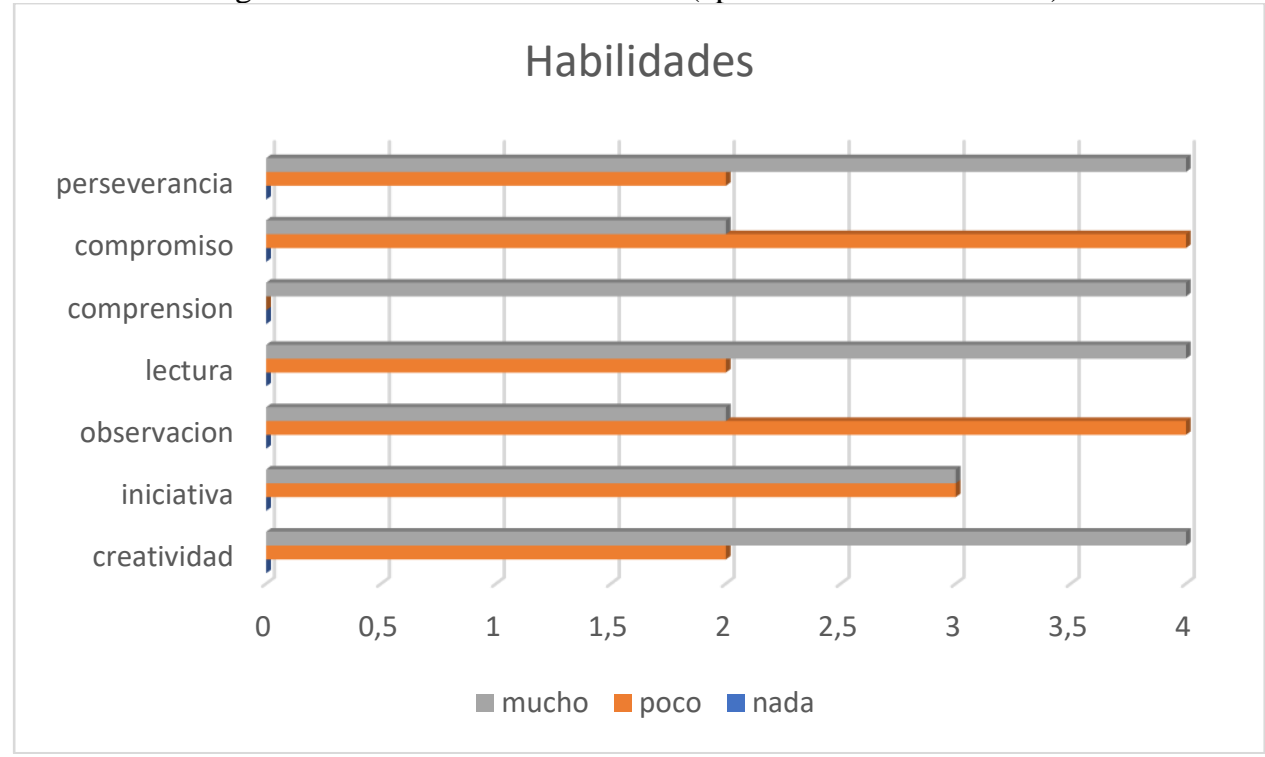


Figura 4: Resultaos de la encuesta (aplicación de competencias transversales).

\section{Competencias tranversales}

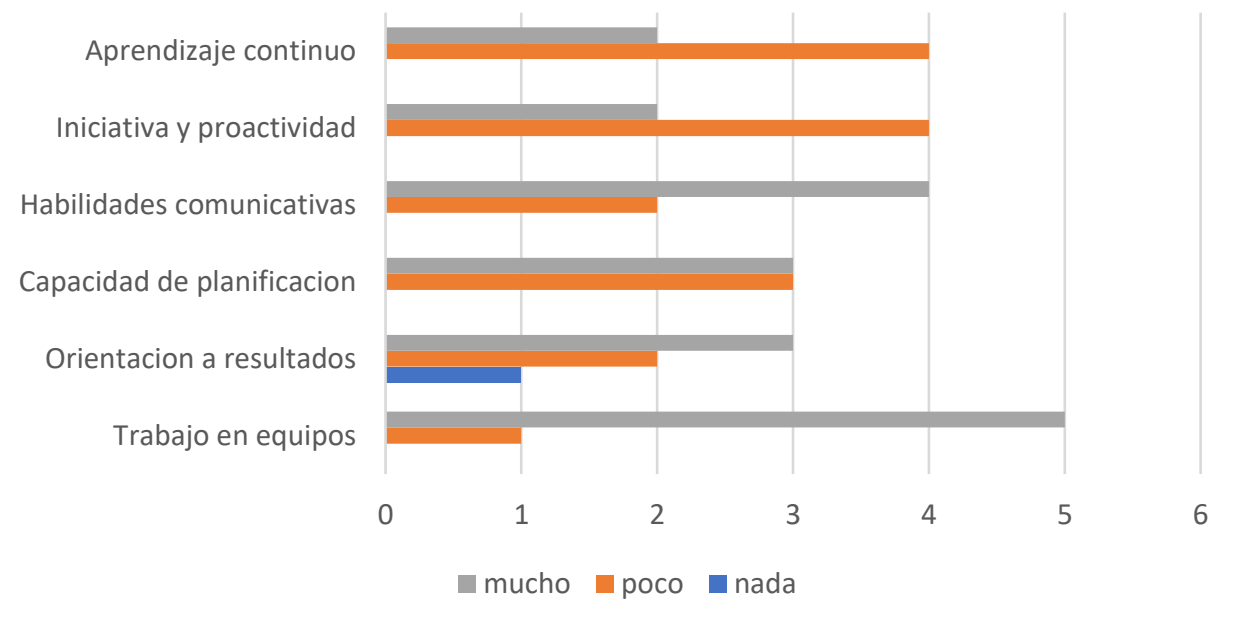

\subsection{DISCUSIÓN}

De acuerdo a los resultados obtenidos después de implementar la metodología ABR, se observa el incremento de alumnos titulados de uno a seis. La metodología ha mostrado ser funcional ya que ha permitido motivar a los alumnos a desarrollar una tesis en la que se apliquen los conocimientos adquiridos en la solución de una problemática real, además que se les ha mostrado que se cuenta con una metodología de trabajo especifica que evita la perdida de tiempos.

De los seis encuestados se puede observar con ayuda de la figura 2 que todos ellos aplicaron satisfactoriamente los conocimientos teorico-practicos durante la realización de su trabajo de tesis.

Las habilidades que se tomaron como variables son: perseverancia, compromiso, comprensión, lectura, observación iniciativa y creatividad; podemos observar que varían de acuerdo a cada uno de los alumnos, con factores externos que no podemos controlar, como puede ser el estado de ánimo. Se puede destacar que la perseverancia y creatividad se han relacionado directamente una con la otra ya que la mayoría de los alumnos buscaron soluciones, más de una vez, lo que en palabras de ellos incentivo su creatividad. Al mismo tiempo se detectaron problemas con la comprensión de la lectura y en especial en un segundo idioma como inglés.

Dentro de las competencias transversales: Aprendizaje continuo, iniciativa y proactividad, habilidades comunicativas, capacidad de planificación, orientación a resultados y trabajo en equipo. Se observa que los alumnos presentaron una fuerte inclinación al dialogo compartiendo conocimientos y llegando a acuerdos con los demás compañeros del laboratorio, por lo que reportan que el trabajo en equipo ha sido fundamental para llegar a la culminación de la tesis. También se obtuvieron bajos resultados en el aprendizaje continuo, algunos alumnos manifestaron que "ya se encontraban saturados de información durante la carrera y seguir aprendiendo durante el desarrollo de la tesis, no era lo que ellos 
buscaban". Por lo que se concluye que aún falta puntualizar información acerca del desarrollo de una tesis en base a la metodología ABR y tener un instrumento de medición que permita evaluar el interés de futuros candidatos.

\section{CONCLUSIONES}

Los efectos de utilizar la metodología ABR, en el desarrollo de tesis de grado han resultado ser positivos ya que su aplicación fue viable, funcional y motivadora, tanto para alumnos como profesores que colaboran en el laboratorio SIRO, la metodología logra integrar conocimientos teórico-prácticos que son parte del plan de estudios de la ingeniería en ciencias de la computación. Al presentarse retos orientados hacia la salud y el medio ambiente, la mayoría de los alumnos presentaron una conducta motivada con el desarrollo y conclusión de la tesis, el alumno aumento sus capacidades comunicativas y de planificación, ya que cada una de las etapas de desarrollo fue calendarizada con la finalidad de no perderse en el camino. La aplicación y desarrollo de las competencias transversales se da de una manera natural, debido a que dentro del laboratorio el alumno tiene la facilidad de interactuar con compañeros que realizan tesis semejantes e incluso algunas de ellas debido a su complejidad se realizan en equipo. La aplicación de esta metodología ha mostrado exigir compromiso y ser un reto para el propio asesor y ha permitido incrementar el interés de los alumnos por realizar tesis para la obtención de grado.

\section{RECONOCIMIENTOS}

Gradecemos a la Vicerrectoría de Investigación y Estudios de Posgrado (VIEP) y a la Facultad de Ciencias de la Computación por el apoyo brindado durante el desarrollo de este trabajo y asistencia al congreso. 


\section{REFERENCIAS}

[1] Benemérita Universidad Autónoma de Puebla. (2019). Licenciatura en Ingeniería en Ciencias de la Computación.Puebla,Pue.:BUAP. Recuperado de: http://cmas.siu.buap.mx/portal_pprd/wb/EDUCATIVA/ingenieria_en_ciencias_de_la_computacion_1

[2] Argudín, Y. (2001). Educación basada en competencias. Educar: revista de educación/nueva época 16

[3] Universia (2012) Qué buscan las empresas en los egresados de ingeniería.México.: Universia México. Recuperado de: https://noticias.universia.net.mx/en-portada/noticia/2012/01/11/904263/que-buscanempresas-egresados-ingenieria.html]

[4] Thornton D.M (2013) for interns, experience isn't always the best teacher. Washington D.C.: The Chronicle of Higher Education. Recuperado de: http://chronicle. Com/article/For-Interns-ExperienceIsnt/143073/.

[5] Observatorio de Innovación educativa del Tecnológico de Monterrey (2015. Reporte Edu Trends. Recuperado de http://eduteka.icesi.edu.co/pdfdir/edutrends-aprendizaje-basado-en-retos.pdf

[6] World Economic Forum (2015). New Vision for Education: Unlocki

[7] Observatorio de Innovación educativa del Tecnológico de Monterrey (2015. Reporte Edu Trends . Recuperado de http://eduteka.icesi.edu.co/pdfdir/edutrends-aprendizaje-basado-en-retos.pdf

[8] Observatorio de Innovación educativa del Tecnológico de Monterrey (2015. Reporte Edu Trends. Recuperado de http://eduteka.icesi.edu.co/pdfdir/edutrends-aprendizaje-basado-en-retos.pdf

[9] Observatorio de Innovación educativa del Tecnológico de Monterrey (2015. Reporte Edu Trends. Recuperado de http://eduteka.icesi.edu.co/pdfdir/edutrends-aprendizaje-basado-en-retos.pdf

[10] Olivares S., López M., Valdez J. Aprendizaje basado en retos: una experiencia de innovación para enfrentar los problemas de salud pública. Educación Médica. Vol. 19, 230-23, recuperado de: https://www.sciencedirect.com/science/article/pii/S157518131730178X https://doi.org/10.1016/j.edumed.2017.10.001

[11] Rosas L., Inclusión de la robótica competitiva en un curso de ingeniería: un proyecto de aprendizaje en retos. Tendencias Educativas. 